\title{
PLANTAS MEDICINAIS POTENCIALMENTE HIPOLIPIDÊMICAS
}

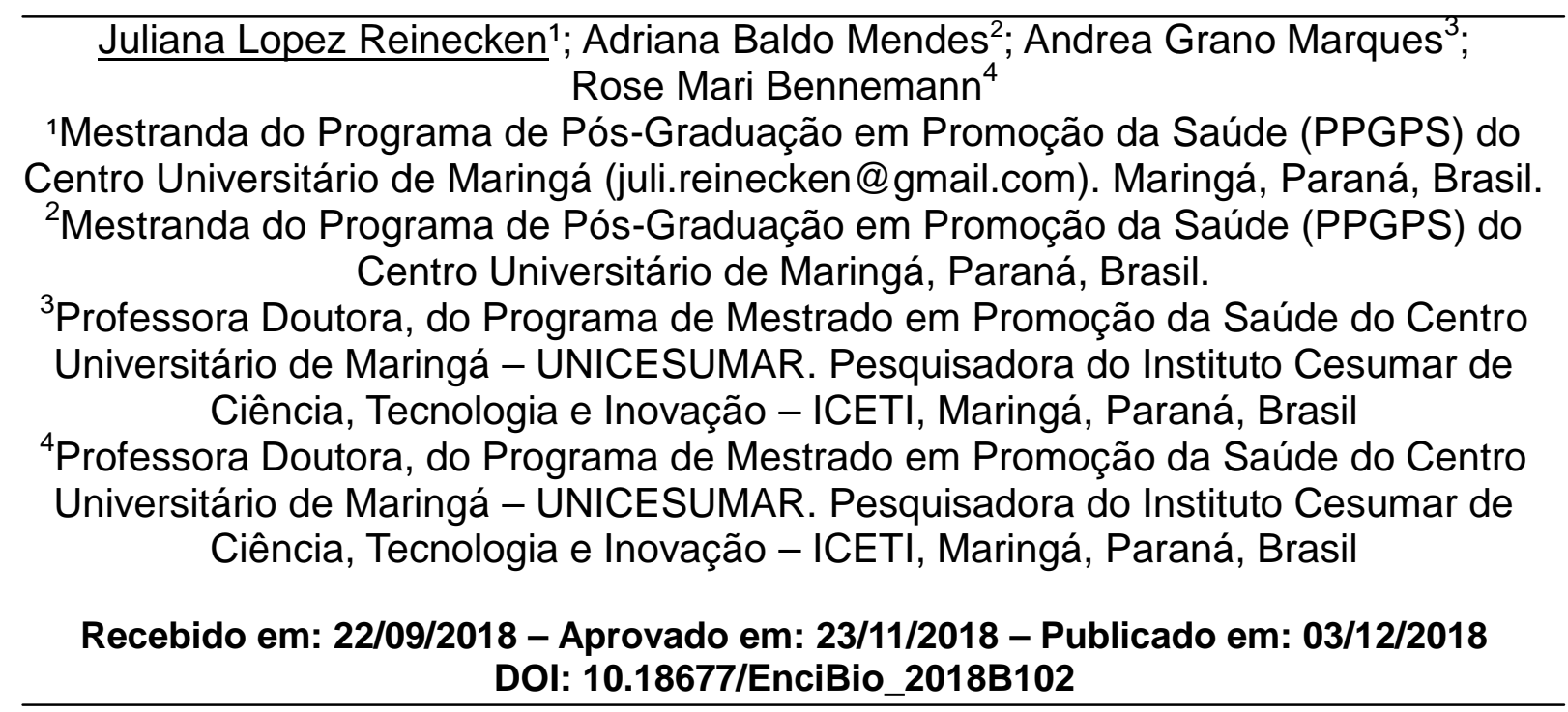

\section{RESUMO}

As doenças cardiovasculares são atualmente consideradas uma das principais causas de mortalidade no mundo, sendo a hiperlipidemia um dos principais fatores de risco para o aumento da prevalência destas doenças. Pesquisas sobre doenças cardiovasculares apontam que o uso de plantas medicinais pode reduzir o risco de surgimento de doenças cardiovasculares. O objetivo da presente pesquisa foi realizar uma revisão na literatura sobre plantas medicinais potencialmente hipolipidêmicas. A revisão foi realizada a partir das bases de dados MEDLINE (Medical Literature Analysis and Retrieval System Online) via PubMed, SciELO (Scientific Electronic Library Online) e BIREME (Centro Latino-Americano e do Caribe de Informação em Ciências da Saúde). Foram selecionados estudos publicados em periódicos nacionais e internacionais, entre os anos de 2007 a outubro de 2017, que tivessem realizado experimentos com plantas medicinais em animais hiperlipidêmicos induzidos a hiperlipidemia e que não isolavam compostos das plantas. A análise incluiu leitura de títulos, resumos e textos completos. Foram encontradas 43 plantas com potencial terapêutico hipolipidêmicos, destas plantas, duas são disponibilizadas no SUS como fitoterápico, Curcuma longa Radix (falsoaçafrão) e Zingiber officinale Roscoe (Gengibre). Diante do exposto, verifica-se que a utilização de plantas medicinais podem ser um importante alternativa no tratamento de doenças cardíacas, que estão iniciando na prática da atenção à saúde brasileira, sugerindo-se mais estudos de comprovação clínica.

PALAVRAS-CHAVE: anti-hiperlipidêmicas, doenças cardíacas, medicina alternativa.

\section{POTENTIALLY HYPOLIPIDEMIC MEDICINAL PLANTS: A LITERARY REVIEW}

\section{ABSTRACT}

Cardiovascular diseases are currently one of the leading causes of mortality worldwide, with hyperlipidemia being a major risk factor for increased disease prevalence. Cardiovascular research indicates that the use of medicinal plants may reduce the risk of cardiovascular disease. The experimental review was carried out in the literature on hypolipidemic medicinal plants. The review was carried out from the 
MEDLINE databases (Analysis and Recovery System of Online Medical Literature) via PubMed, SciELO and BIREME. We have found studies published in national and international journals between 2007 and October 2017, which were carried out with medicinal plants in hyperlipidemics induced in hyperlipidemia and that did not isolate the plants. An analysis included the reading of titles, abstracts and full texts. There were 43 plants with hypolipidemic therapeutic potential, both of which are available in SUS as herbal medicine, Curcuma longa Radix (false saffron) and Zingiber officinale Roscoe (Ginger). In view of the above, it is verified that the use of medicinal plants can be an important alternative in the treatment of heart diseases, which are beginning in the practice of Brazilian health care, suggesting further studies of clinical evidence.

KEYWORDS: antihyperlipidemic, cardiac diseases, alternative medicine.

\section{INTRODUÇÃO}

As doenças cardiovasculares, incluindo a doença cardíaca coronária e aterosclerose, são as principais causas de morte reconhecidas mundialmente. Segundo a Organização Mundial de Saúde, até o ano de 2030, mais de 23 milhões de pessoas morrerão anualmente de doenças cardiovasculares, ocorrendo a maior parte em países de baixa e média renda (OMS, 2016). Os fatores para o desenvolvimento destas doenças são inúmeros, as dietas calóricas ricas em ácido graxos saturados e colesterol, fatores genéticos, idade, estilo de vida inadequado como o sedentarismo, desempenham um papel importante ao desenvolvimento das doenças cardiovasculares. Todavia, altos níveis de triglicerídeos, colesterol e a lipoproteína de baixa densidade (LDL), são considerados como importantes fatores de risco para o desenvolvimento das doenças cardiovasculares, que também se correlacionam com a hipertensão, doença coronariana, aterosclerose, dislipidemia, entre outros (KAMAL et al., 2017; RADDY et al., 2015). A Sociedade Brasileira de Cardiologia, em sua última diretriz, reafirmou que níveis elevados de colesterol e triglicerídeos, são os principais fatores de riscos associados a aterosclerose (FALUDI et. al., 2017).

Além disso, distúrbios do metabolismo lipídico também estão intimamente ligados ao aumento do estresse oxidativo, isto porque os radicais livres, estão envolvidos no desenvolvimento inicial e progressão da aterosclerose de indivíduos hiperlipidêmico através do dano oxidativo causados pelas lipoproteínas presentes no sangue. Este estresse oxidativo nestes indivíduos, aumenta ainda mais o risco de doenças cardiovasculares, isto devido a oxidação lipídica que é gerada principalmente pelo LDL oxidado, esta lipoproteína está envolvido em diversos eventos que levam a placa de ateroma, desenvolvendo assim a aterosclerose (SINGH et al., 2016).

A hiperlipidemia é um distúrbio considerado comum entre a população mundial, afetando aproximadamente $25 \%$ dos adultos nos países desenvolvidos. Estudos em animais, tem demonstrado que a hiperlipidemia está ligada com a esteatose hepática, visto que níveis elevados de triglicerídeos podem acumular-se no fígado. Também este distúrbio está relacionado com a obesidade, que é consequente devido à alta ingestão de gordura, sedentarismo e fatores genéticos (HAN et al., 2015).

Métodos farmacológicos mais comuns para o controle da hiperlipidemia são utilizados como as estatinas, fibratos e niacina. Estes fármacos possuem efeitos anti-hiperlipêmicos, reduzindo os níveis de LDL sérico e inibem a síntese de colesterol no fígado (HAN et al., 2015; SINGH et al., 2016). Entretanto, as limitações 
que existem no uso destes medicamentos, assim como os efeitos colaterais, tais como insuficiência hepática e renal, diarreia, hipoglicemia, fazem estes medicamentos intoleráveis para alguns pacientes que necessitam administrá-los. Desta forma, a busca por novas alternativa que visam encontrar novos compostos que aumentem a eficácia e também diminuam a sua toxicidade, evolui cada vez mais, incentivando no desenvolvimento de novas pesquisas que busquem alternativas em compostos naturais (ZANG et al., 2016).

As plantas medicinas são de grande importância na saúde primaria, além disso são importantes para o desenvolvimento de novas drogas terapêuticas. Atualmente estima-se que $40 \%$ dos medicamentos disponíveis originam de fontes naturais, dos 252 fármacos considerados essenciais $11 \%$ possui origem de plantas consideradas medicinais (BRASIL, 2016). Estas são tradicionalmente fonte natural mais antiga, que possuem pouco ou nenhum tipo de efeito colateral, tornando-se de grande valia para a sua exploração e pesquisas com o intuito de identificar seus potenciais terapêuticos (RADDY et al., 2015). Plantas medicinais que possui atividade terapêutica anti-hiperlipidêmicos, já reconhecidas, determinadas e validadas, tornam-se importante alternativa de baixo custo para o sistema da saúde. Além disso, os seus efeitos colaterais na grande maioria são menores, se comparados as formas farmacológicas sintéticas (PEREIRA et al., 2015). Diante disto, este estudo tem como objetivo identificar as plantas medicinais potencialmente hipolipidêmicas. que possuem potenciais terapêuticos anti-hiperlipidêmicos, e desta forma contribuir para o desenvolvimento de mais pesquisa que otimizem alternativas naturais devido ao seu baixo custo e efeitos tóxicos menores que os medicamentos sintéticos.

\section{MATERIAL E MÉTODOS}

O presente trabalho caracteriza-se como uma revisão da literatura. Foram selecionados artigos científicos publicados entre 2007 a outubro de 2017, nas bases de dados MEDLINE (Medical Literature Analysis and Retrieval System Online) via PubMed, SciELO (Scientific Electronic Library Online) e BIREME (Centro LatinoAmericano e do Caribe de Informação em Ciências da Saúde), considerando todos os artigos científicos disponibilizados gratuitamente nas bases de dados, com texto completo independentemente do idioma e o fator de impacto da revista. Foram selecionados estudos que realizaram experimentos com plantas medicinais em animais hiperlipidêmicos, com o intuito de identificar a eficácia do tratamento com plantas medicinais.

Os descritores utilizados na consulta das referidas bases de dados, foram as seguintes combinações de palavras: "Plantas medicinais and dislipidemias", "antihyperlipidemic plants" e "hipocolesterolemia". Esta busca resultou inicialmente nos seguintes números: 298 artigos científicas na MEDLINE, 264 na BIREME e 08 na Scielo, totalizando 570 artigos científicos publicados entre 2007 e 2017.

A busca dos artigos de interesse foi desenvolvida em três etapas: primeiramente, foram lidos todos os títulos dos artigos encontrados nas bases de dados com os descritores utilizados, sendo excluídos artigos duplicados e que não se relacionavam em experimentos com plantas medicinais em animais com hiperlipidemia. Após esta análise, partiu-se na leitura dos resumos, dentre os quais foram excluídos os que isolaram compostos químicos das plantas e que não utilizaram animais hiperlipidêmicos. Finalmente, foram lidos e avaliados os textos na íntegra, a fim de verificar a comprovação do potencial anti-hiperlipidêmicos das plantas medicinais. 


\section{RESULTADOS E DISCUSSÃO}

Neste estudo foi realizado um levantamento bibliográfica, em que foram selecionados 49 estudos que atendiam os critérios de inclusão. A partir desta etapa, analisou-se o resumo, em que foi possível observar as plantas que obtiveram efeitos benéficos com ação hipolipemiante (Quadro 1), sendo que apenas uma espécie foi obtida no Brasil, Camellia sinensis L. (Chá verde). A maioria dos estudos obteve as plantas medicinais na Índia correspondente a 30\%, seguida da China com 19\% e $10 \%$ no Irã. Das 43 plantas com potencial terapêutico hipolipidêmicos, duas são disponibilizadas no SUS como fitoterápico, Curcuma longa Radix (falso-açafrão) e Zingiber officinale Roscoe (Gengibre) (BRASIL, 2009). Também a grande parte dos estudos, utilizaram a planta com um todo para a análise da pesquisa correspondente a $19 \%$, seguido da utilização do seu fruto com $14 \%$ e a semente $13 \%$.

QUADRO 1. Artigos selecionados após a análise do título que apresentaram experimentos com plantas que possui potencial terapêutico anti-hiperlipidêmico.

\begin{tabular}{|c|c|c|c|c|}
\hline Lista das espécies & Artigos & $\begin{array}{c}\text { Nome } \\
\text { Popular }\end{array}$ & Região & $\begin{array}{c}\text { Parte utilizada } \\
\text { na pesquisa }\end{array}$ \\
\hline $\begin{array}{l}\text { Actinidia deliciosa } \\
\text { Liang e Ferguson }\end{array}$ & 1 & Kiwi & China & Fruto \\
\hline $\begin{array}{l}\text { African mistletoe } \\
\text { Loranthaceae }\end{array}$ & 1 & Viscum album & Nigéria & Planta \\
\hline $\begin{array}{l}\text { Allium ampeloprasum } \\
\text { Linnaeus }\end{array}$ & 1 & Alho-poró & Irã & Planta \\
\hline Amaranthus spinosus L. & 1 & $\mathrm{~N} / \mathrm{D}$ & Índia & Caule \\
\hline $\begin{array}{l}\text { Artemisia iwayomogi } \\
\text { Haninjin }\end{array}$ & 1 & $\mathrm{~N} / \mathrm{D}$ & Coreia & Planta \\
\hline $\begin{array}{l}\text { Boswellia serrata } \\
\text { Triana e Planch }\end{array}$ & 1 & $\begin{array}{l}\text { Boswellin, } \\
\text { salai guggal, } \\
\text { olíbano, } \\
\text { incenso, } \\
\text { olibano }\end{array}$ & Índia & Rezina \\
\hline Camellia sinensis L. & 1 & Chá verde & China & Flor \\
\hline Caryocar coriaceum Wittm. & 1 & Pequi & Brasil & Fruto \\
\hline Citrullus colocynthis L. & 1 & Colocíntida & Irã & $\begin{array}{l}\text { Semente } \\
\text { Polpa }\end{array}$ \\
\hline Clitoria ternatea L. & 1 & $\begin{array}{c}\text { Clitóris, } \\
\text { Clítoris, } \\
\text { Cunhã, Feijão- } \\
\text { borboleta, } \\
\text { Ervilha- } \\
\text { borboleta, } \\
\text { Rainha-azul }\end{array}$ & Índia & Semente \\
\hline Cornus mas L. & 1 & \begin{tabular}{|c|} 
Corniso, \\
Cerejas \\
corneínas \\
\end{tabular} & N/D & Fruto \\
\hline Cucumis melo L. & 1 & Melão & Índia & Fruto \\
\hline $\begin{array}{l}\text { Cucurbita: } \\
\text { Cucurbita maxima } \\
\text { Duchesne }\end{array}$ & 2 & Abóbora & $\begin{array}{l}\text { Cuba e } \\
\text { México }\end{array}$ & Semente \\
\hline
\end{tabular}




\begin{tabular}{|c|c|c|c|c|}
\hline $\begin{array}{l}\text { Cucurbita moschata } \\
\text { Duchesne ex Poir. }\end{array}$ & & & & \\
\hline Curcuma longa L. & 1 & $\begin{array}{c}\text { falso-açafrão, } \\
\text { açafrão-da- } \\
\text { terra, }\end{array}$ & Coreia & Planta \\
\hline Cydonia oblonga Miller & 1 & Marmeleiro & China & $\begin{array}{c}\text { Folhas } \\
\text { Fruto }\end{array}$ \\
\hline $\begin{array}{l}\text { Dioscorea nipponica } \\
\text { Makino }\end{array}$ & 1 & $\mathrm{~N} / \mathrm{D}$ & China & Rizoma \\
\hline $\begin{array}{l}\text { Dysoxylum binectariferum } \\
\text { Hiern. }\end{array}$ & 1 & N/D & Índia & $\begin{array}{c}\text { Planta - } \\
\text { Composto } \\
\text { Isolado }\end{array}$ \\
\hline $\begin{array}{l}\text { Flacourtia indica } \\
\text { Burman F. }\end{array}$ & 1 & $\begin{array}{l}\text { Ameixa do } \\
\text { governador }\end{array}$ & Índia & Folha \\
\hline $\begin{array}{l}\text { Livistona chinensis Jacq. } \\
\text { Livistona decipiens Becc. }\end{array}$ & 2 & $\begin{array}{l}\text { Palmeira de } \\
\text { Leque ou } \\
\text { Falsa Latânia }\end{array}$ & Egito & Fruto \\
\hline $\begin{array}{l}\text { Melastoma malabathricum } \\
\text { L. }\end{array}$ & 1 & $\begin{array}{c}\text { Rhododendron } \\
\text { indiano }\end{array}$ & Índia & Folha \\
\hline $\begin{array}{l}\text { Pandanus tectorius } \\
\text { Parkinson }\end{array}$ & 1 & $\begin{array}{l}\text { Pandano- } \\
\text { havaiano }\end{array}$ & China & Fruto \\
\hline Panicum repens $L$. & 1 & capim-torpedo & Egito & $\begin{array}{c}\text { Raiz } \\
\text { Rizoma }\end{array}$ \\
\hline Pinus eldarica Medw & 1 & Pinheiro & Irã & Semente \\
\hline $\begin{array}{l}\text { Pinus koraiensis } \\
\text { Siebold e Zucc. }\end{array}$ & 1 & Pinheiro & Coreia & Folhas \\
\hline Pistacia khinjuk Stocks & 1 & $\mathrm{~N} / \mathrm{D}$ & Paquistão & Folhas \\
\hline Potentilla chinesis Ser. & 1 & $\mathrm{~N} / \mathrm{D}$ & China & $\begin{array}{l}\text { Planta - } \\
\text { Composto } \\
\text { Isolado }\end{array}$ \\
\hline Protorhus longifolia Bernh. & 1 & N/D & $\begin{array}{l}\text { África do } \\
\text { Sul }\end{array}$ & Caule \\
\hline $\begin{array}{l}\text { Psacalium decompositum } \\
\text { A.Gray }\end{array}$ & 1 & Matarique & México & Raiz \\
\hline Pteris multifida Poiret & 1 & $N / D$ & China & Planta \\
\hline $\begin{array}{l}\text { Rehmannia glutinosa } \\
\text { Gaertn. }\end{array}$ & 1 & $N / D$ & China & Planta \\
\hline Salacia reticulata Wight & 1 & $\begin{array}{l}\text { Kothala } \\
\text { Himbutu }\end{array}$ & Índia & $\begin{array}{c}\text { Raiz } \\
\text { Hastes } \\
\text { Folha }\end{array}$ \\
\hline Salvia officinalis L. & 1 & Salvia & Irã & Folha \\
\hline $\begin{array}{l}\text { Semecarpus anacardium } \\
\text { L. }\end{array}$ & 1 & $\begin{array}{l}\text { Caju, vitex, } \\
\text { chasteberry }\end{array}$ & Índia & Semente \\
\hline Shorea roxburghii G. Don. & 1 & Phayom & Tailândia & Casca \\
\hline Terminalia Arjuna Roxb. & 1 & N/D & Índia & Planta \\
\hline Terminalia chebula Retz. & 1 & $\mathrm{~N} / \mathrm{D}$ & Índia & Casca \\
\hline Ulva lactuca L. & 1 & Alface do Mar & Índia & Planta \\
\hline Vaccinium myrtillus L. & 1 & Mirtilo & Irã & Fruta \\
\hline Vigna mungo L. & 1 & feijão-preto & Índia & Semente \\
\hline
\end{tabular}

ENCICLOPÉDIA BIOSFERA, Centro Científico Conhecer - Goiânia, v.15 n.28; p. $1293 \quad 2018$ 


\begin{tabular}{|l|l|l|l|l|}
\hline Withania coagulans Dunal & 1 & N/D & Índia & Planta \\
\hline Zingiber officinale Roscoe & 2 & Gengibre & China & Rizoma \\
\hline
\end{tabular}

N/D: não definido pelo artigo.

Esta análise dos resumos decorreu na seleção de 18 artigos, por serem pesquisas com experimentos in vivo em animais induzidos a hiperlipidemia e que não isolavam compostos das plantas. Após a avaliação dos artigos, o estudo selecionou e concentrou-se em 10 artigos de interesse $(1,7 \%$ do total de artigos publicados nas três bases de dados).

No Quadro 2, é possível identificar que os anos de publicação foram distribuídos em um artigo para cada ano de 2007, 2009, 2010, 2016, 2017 e cinco artigos foram publicados em 2015. Outro item analisado, foram quais as principais atividades terapêuticas encontradas nas plantas medicinais, uma vez que além de terem potenciais anti-hiperlipidêmicas, apresentaram ações antioxidante, remoção dos radicais livres na corrente sanguínea, anti-inflamatório, efeitos estes que ocasionam fatores cardioprotetor. Também identificamos nestes artigos selecionados, que a Índia e a China são os que mais apresentam pesquisas com plantas medicinais. Isto devido a decorrência aos seus contextos históricos entre estes países, sendo uma prática milenar, utilizada até os dias atuais (FIRMO et al., 2011). Apesar destes achados, não podemos deixar de destacar que o Brasil é o país em destaque pela sua biodiversidade considerada a mais rica, o que implica em diversas fontes terapêuticas que ainda não foram totalmente pesquisadas, estima-se que somente $15 \%$ das espécies vegetais tenham sido pesquisadas (ZAGO, 2018).

QUADRO 2. Total de artigos analisados e selecionados integralmente.

\begin{tabular}{|c|c|c|c|c|}
\hline $\begin{array}{c}\text { Planta } \\
\text { analisada }\end{array}$ & Ano & $\begin{array}{c}\text { País da } \\
\text { IES }\end{array}$ & Revista & Atividade-terapêutica \\
\hline $\begin{array}{c}\text { Flacourtia } \\
\text { indica } \\
\text { Burm. F. }\end{array}$ & 2015 & Índia & $\begin{array}{c}\text { Journal of Basic and } \\
\text { Clinical Physiology and } \\
\text { Pharmacology }\end{array}$ & $\begin{array}{c}\text { Hipolipidêmicos e } \\
\text { antioxidante }\end{array}$ \\
\hline $\begin{array}{c}\text { Caryocar } \\
\text { coriaceum } \\
\text { Wittmack }\end{array}$ & 2016 & Brasil & $\begin{array}{c}\text { Journal of } \\
\text { Ethnopharmacology } \\
\text { Panicum } \\
\text { repens L. }\end{array}$ & $\begin{array}{c}\text { Zeitschrift für } \\
\text { Naturinflamatório, } \\
\text { hipolipidêmicos e } \\
\text { cardioprotetor }\end{array}$ \\
\hline $\begin{array}{c}\text { Clitoria } \\
\text { ternatea L. } \\
\text { e Vigna } \\
\text { mungo L. }\end{array}$ & 2009 & Egito & Índia & Anti-hiperlipidêmica. \\
\hline $\begin{array}{c}\text { Pteris } \\
\text { multifida } \\
\text { Poir. }\end{array}$ & 2010 & China & Pharmaceutical Biology & $\begin{array}{c}\text { Antioxidante natural nos } \\
\text { tecidos do fígado e } \\
\text { hipolipidêmicos }\end{array}$ \\
\hline $\begin{array}{c}\text { Terminalia } \\
\text { chebula } \\
\text { Reitz. }\end{array}$ & 2015 & Índia & Pharmaceutical Biology & $\begin{array}{c}\text { Anti-hiperlipidêmico, } \\
\text { diminui da absorção de } \\
\text { excresterol e aumenta a de compostos } \\
\text { de nitrogênio, esteroides } \\
\text { neutros e ácido biliar nas } \\
\text { fezes. }\end{array}$ \\
\hline
\end{tabular}




\begin{tabular}{|c|c|c|c|c|}
\hline $\begin{array}{c}\text { Pistacia } \\
\text { khinjuk } \\
\text { Stocks }\end{array}$ & 2017 & Paquistão & $\begin{array}{c}\text { Biomedicine \& } \\
\text { Pharmacotherapy }\end{array}$ & Hlipolipidêmicos no sangue. \\
\hline $\begin{array}{c}\text { Citrullus } \\
\text { colocynthi } \\
\text { s L. }\end{array}$ & 2007 & Irã & $\begin{array}{c}\text { Revista Brasileira de } \\
\text { Farmacognosia }\end{array}$ & Anti-hiperlipidêmicas \\
\hline $\begin{array}{c}\text { Cydonia } \\
\text { oblonga } \\
\text { Miller }\end{array}$ & 2015 & China & $\begin{array}{c}\text { Journal of } \\
\text { Ethnopharmacology }\end{array}$ & $\begin{array}{c}\text { Regula o metabolismo } \\
\text { dos lipídios, removem os } \\
\text { radicais livres de } \\
\text { oxigênio e melhoram os } \\
\text { potenciais antioxigenicos }\end{array}$ \\
\hline $\begin{array}{c}\text { Artemisia } \\
\text { iwayomogi } \\
\text { Haninjin } e \\
\text { Curcuma } \\
\text { longa L. }\end{array}$ & 2015 & Coreia & Ethnopharmacology & Anti-hiperlipidêmicos \\
\hline
\end{tabular}

Fonte: dados da pesquisa, 2018.

A maioria dos estudos descreveu efeitos hiperlipidemicos nos animais que compuseram a amostra da pesquisa com dieta elevada em gordura (ARTIGOS: ELTANTAWY et al., 2014; WANG et al., 2010; REDDY et al., 2015; HAN et al., 2015; KAMAL et al., 2017). Identificando desta forma a importância dos hábitos saudáveis, incluindo a alimentação, exercícios físicos e diminuição do estresse. Importante ressaltar que a hiperlipidemia acomete outras doenças/distúrbios que estão intimamente ligadas as doenças cardíacas como as dislipidemias, aterosclerose, acidente vascular cerebral, infarto agudo do miocárdio, diabetes, obesidade, fatores genéticos, entre outros (FALUDI et. al., 2017).

Dentre os estudos selecionados não houve repetição de plantas analisadas e, também, apresentaram a análise de uma ou duas plantas por artigo. Com base nesses estudos serão descritos brevemente os potenciais de cada planta medicinal descrita nos artigos selecionados.

A planta Flacourtia indica, obtida em uma árvore pequena nativa da Índia, possui valores medicinais reconhecidas mundialmente para o tratamento de diversas doenças. Na África esta planta é popularmente utilizada entre os curandeiros tradicionais para o manejo da malária e complicações relacionadas a esta doença (SINGH et al., 2017). Além de outras doenças como a icterícia, cólera, diabetes e atividade anti-hiperlipidêmica que o estudo selecionado nesta revisão identificou. Neste estudo, os ratos foram induzidos por Triton a obter efeitos hiperlipidêmicos, os benefícios observados neste estudo referente ao extrato $F$. indicam, foram sobre o metabolismo lipídico endógeno, obteve-se uma diminuição no índice aterogênico e um aumento da relação do LDL/HDL, isso é, havendo uma diminuição dos níveis do LDL e um aumento nos níveis do HDL. A planta também apresentou efeitos antioxidantes, dado que a mesma apresentou atividade de eliminação de radicais livres. $O$ estudo destaca que estes benefícios podem ter ocorrido devido a presença de flavonoides na planta, que possuem atividades antidislipidémicas e antioxidantes (SINGH et al., 2016).

A espécie Caryocar coriaceum árvore brasileira popularmente conhecida como "pequizeiro", é encontrada na região brasileira do extremo norte e parte do nordeste, desempenhando um importante papel socioeconômico nesta região. O seu 
fruto é utilizado na elaboração de alimentos e na preparação de molhos e especiarias comuns da região. No estudo selecionado, foi identificado potenciais positivos da Caryocar coriaceum em animais que foram induzidos a hiperlipidemia através do fármaco Triton. O estudo observou redução tanto na hipercolesterolemia, quanto a hipertrigliceridemia, relacionando este efeito à inibição da HMG-CoA redutase (enzima que está relacionada na biossíntese do colesterol, que é inibida por estatinas), ou por ativação de LPL (enzima relacionada à hidrólise de triglicerídeos e VLDL). O estudo também não identificou toxicidade do "pequi" nas doses testadas, provavelmente por decorrência as características químicas encontradas como carotenoides, compostos fenólicos, incluindo flavonoides, taninos e alfa-tocoferol na polpa de pequi (DE FIGUEIREDO et al., 2016). Outro estudo destaca a importância desta espécie em diversas outras patologias como no tratamento de queimaduras, gripes, úlceras cutâneas, inflamação na pele, infecções broncopulmonares, dores osteomusculares. Achados fitoquímicos identificam diversos ácidos graxos no óleo do "pequi", como o ácido oleico, que assim como o azeite de oliva e demais óleos vegetais, possuem esta característica que são utilizados no tratamento da hiperlipidemia, potencializando os efeitos cardioprotetores (KERNTOPF et al., 2013).

Panicum repens L. (Poaceae) conhecido também como "capim-torpedo", nativo da África e Ásia. Trata-se de uma planta perene com presença de rizomas longos e rastejantes (TOSATI; SCHEFFER-BASSO, 2007). É principalmente uma erva daninha dos solos úmidos e costeiros, embora também cresça em solos pesados de montanha. Não possui nenhum relato sobre suas atividades biológicas de extratos de $P$. repens, desta forma, no estudo selecionado, realizou um experimento para identificar os efeitos anti-hiperlipidêmicos desta planta. Para isto, os animais foram submetidos a elevado consumo de colesterol e posteriormente foram tratados com extrato de Panicum repens L., o estudo obteve resultados relevantes principalmente na manutenção do metabolismo dos lipídios no sangue, foi identificado que houve uma diminuição da absorção de colesterol e um aumento na excreção de compostos de nitrogênio, esteroides neutros e ácido biliar nas fezes, tendo desta forma não só efeitos anti-hiperlipidêmico (EL-TANTAWY et al., 2014).

As plantas Clitoria ternatea e Vigna mungo, pertencentes a família Fabaceae, foram citadas no estudo de Solaki e Jain 2010, como plantas comumente utilizadas para efeitos anti-hiperlipidêmico na Índia. São plantas consideradas leguminosas contendo uma fonte rica em proteínas alimentares, o que sugere plantas que podem favorecer a diminuição dos níveis lipídios no sangue. O estudo selecionado, foi realizado com animais hiperlipidêmicos induzidos por poloxâmer 407 e também por dieta. Os resultados da pesquisa foram comparados com o fármaco atorvastatina e gemfibrozila, que foram os padrões de referência para a pesquisa. $O$ estudo demonstrou alta eficiência entre estas duas plantas em relação a potenciais antihiperlipidêmico, redução na oxidação lipídica, distúrbio este que se elevado favorece a aterosclerose, além de demonstrar melhora no estado antioxidante do fígado, otimizando as atividades de diversas enzimas (SOLANKI; JAIN, 2010). Em outro estudo que avaliou efeitos hiperlipidêmicos da Clitoria ternatea, considera-se outros atributos a esta planta com ampla atividade farmacológica incluindo antimicrobiano, antipirético, anti-inflamatório, antiasmático, hepatoprotetor, analgésico, diurético, anestésico local, inseticida, inibidor de agregação plaquetária e com propriedades relaxantes. Além destes, o estudo identificou efeitos da planta importantes para o tratamento da diabetes como efeitos que ajudam na regeneração do pâncreas e potenciais antidiabéticos e anti-hiperlipidêmicas (VERMA; ITANKAR; ARORA, 2013). 
Já a planta Vigna mungo, em um outro estudo, identificou-se como um importante aliado na síndrome metabólica, síndrome considerada como uma epidemia no mundo, isto devido a essa síndrome aumentar a prevalência no desenvolvimento de doenças cardíacas. No estudo, os efeitos da planta foram comparados com o fármaco atorvastatina, obtendo efeitos melhores que o fármaco, em razão de a Vigna mungo não ter apresentado efeitos colaterais, sugerindo-se como um importante aliado no tratamento da síndrome metabólica (KAUR; PATEL, 2015).

Pteris multifida (Pteridaceae), amplamente distribuída na China, comumente utilizado na medicina tradicional chinesa para o tratamento de hepatite, disenteria bacteriana, enterite, hematêmese, hematúria, amigdalite, parotidite e inflamação na pele. A planta possui uma série de compostos com bioativos, antitumoral de diterpenoides ent-kaurane e pterosinas (WANG et al., 2010). No estudo selecionado os ratos foram induzidos à uma dieta elevada de colesterol para a indução da hiperlipidemia, os animais que foram tratados com a Pteris multifida obtiveram efeitos anti-hiperlipidêmico muito definido, além de componentes benéficos na manutenção do metabolismo dos lipídios no sangue dos animais. Os mecanismos para a obtenção destes efeitos, foram devido a diminuição da absorção do colesterol e o aumento da excreção de compostos de nitrogênio, esteroides neutros e ácido biliar nas fezes (WANG et al., 2010).

Terminalia chebula Retz. pertencente à família Combretaceae, conhecida por muitos países asiáticos como a Índia e o Paquistão, e também em países africanos (REDDY et al., 2015). Além de seus efeitos anti-hiperlipidêmicos, tem sido cientificamente comprovada para promover imunidade, saúde e longevidade. Os extratos orgânicos e aquosos de $T$. chebula exibem antioxidantes, antimicrobianos, antianafiláticos, antidiabéticos, antimutagênicos, anticancerígenos, apoptóticos, anticaries, antifúngicas e atividades antivirais. (SARABHAI, SHARMA, CAPALASH, 2013). No estudo selecionado nesta pesquisa, os animais foram submetidos a uma elevada dieta de colesterol para a obtenção da hiperlipidemia. Os grupos foram tratados com o fármaco atorvastatina, que foi considerado como um controle positivo para uma comparação adequado sobre os efeitos anti-hiperlipêmico que foram comparados com o extrato de Terminalia chebula. O estudo demonstrou ação antihiperlipidêmica principalmente na dose administradas pelos ratos de $600 \mathrm{mg} / \mathrm{kg}$. Foi observado que os extratos com a doses administradas foram desprovidos de grandes efeitos tóxicos. Além disso, houve também uma redução significativa no nível de glicose no sangue, podendo o mesmo estabelecer como um importante alternativa para o tratamento da hiperlipidemia (REDDY et al., 2015). Outros benefícios foram descritos no estudo de Jokar e colaboradores, como efeito antidiabético e renoprotetor, atividade cardiotônica no aumenta a força das contrações e do débito cardíaco, sem alterar a frequência cardíaca, contém agentes antioxidantes, incluindo quercetina e vitamina $\mathrm{C}$, que funcionam contra a neurodegeneração induzida pelo estresse oxidativo, também identificou-se atividade de eliminação de radicais livres in vitro e atividade antioxidante de redução férrica (JOKAR et al., 2016).

A Pistacia khinjuk considerada uma das grandes espécies do gênero Pistacia na família Anacardiaceae, que está presente em alguns países da Ásia, como Irâ, Iraque e Síria, é uma planta comumente utilizada no tratamento de doenças relacionadas a pele e a digestão (SODEIFIAN et al., 2015). As espécies Pistacia normalmente apresentam efeitos antiaterogênicos, antiinflamatórios, antioxidantes, antimicrobianos, hipoglicemicos, diurético em tratamentos para a infecção urinaria, efeitos protetores contra o câncer, sendo as suas folhas ricas em 
olhos essenciais. O estudo selecionado avaliou efeitos anti-hiperlipidêmicos desta planta, em ratos que foram administrados com dieta de alta gordura. O resultado do estudo foi benéfico tanto em doses baixas como em doses elevados do extrato da planta, obtendo diminuição do nível do colesterol, triglicerídeos e LDL (KAMAL et al., 2017). Outros estudos que analisaram as composições químicas do óleo do fruto desta planta, identificaram a presença de tocotrienóis, conhecidos por terem atividade antioxidante, podendo ter benefícios na proteção da pele contra a peroxidação de radicais livres, bem como propriedades cardioprotetoras, neuroprotetoras e anticancerígenas, sugerindo desta forma a utilização do óleo na indústria farmacêutica (ASNAASHARI et al., 2015).

Citrullus colocynthis descrito na farmacopeia entre os medicamentos homeopáticos mais utilizados, a mesma possui indicações para cólicas abdominais e diarreias com cólicas doloridas (ANVISA, 2011; ANVISA, 2017). É uma planta de climas tropicais, comumente encontrada na Ásia tropical e África, pertence às espécies do gênero Citrullus da família das cucurbitáceas, que geralmente consiste de um grande número de variedades. A sua polpa é esponjosa branca, possui glicosídeos sendo efetivo como laxante, já as suas sementes são comestíveis contendo óleo e ácidos insaturados (NEHDI et al., 2013). No estudo selecionado, foi observado efeitos da polpa e da semente desta planta em coelhos induzidos a hiperlipidemia por uma dieta rica em colesterol. O estudo obteve resultados positivos sobre efeitos no perfil lipídico do sangue, obtendo uma redução significativa do colesterol sérico total e LDL-C em grupos que foram tratados com os extratos tanto da semente como da polpa, este resultado foi relacionado pela presença de saponinas presente na Citrullus colocynthis, sabe-se que plantas contendo saponinas possui também efeitos anti-hiperlipidêmicos (ZAMANI et al., 2007).

Cydonia oblonga Miller é uma planta da família Rosaceae, comumente utilizada na medicina tradicional chinesa (ZHOU et al., 2014). É constituída por baixo teor de gordura e possui uma fonte importante de ácidos orgânicos, fibras brutas, açúcares e minerais como fósforo, potássio e cálcio, assim como compostos que possui atividade antioxidante como os fenólicos, assim como propriedades hipoglicemiantes, anti-inflamatórias, antimicrobianas, anticarcinogênicas, antialérgicas e antiulcerativas, atuando também como um importante cardioprotetor (LEGUA et al., 2013). No estudo selecionado foi realizado uma pesquisa com ratos hiperlipidêmicos, os mesmos foram submetidos ao tratamento com extrato de flavonóides da fruta da Cydonia oblonga Miller, no estudo observou-se níveis significativamente mais baixos de triglicerídeos e LDL-C, enquanto que o HDL-C aumentou, indicando que os flavonóides totais de frutas e folhas desta planta podem regular os lipídios em ratos hiperlipidêmicos. Quanto aspectos a lesões hepáticas obtido no estudo, os ratos tratados com o extrato Cydonia oblonga Miller, obtiveram os marcadores bioquímicos AST e ALT com níveis menores, identificando diminuição dos danos hepáticos. Também o estudo identificou aspectos favoráveis na remoção dos radicais livres, considerando-se desta forma potenciais antioxigenicos. (UMAR et al., 2015). Em outra pesquisa utilizou-se a mesma planta para verificar potenciais na diminuição da pressão arterial, na qual foi constatado uma interação com o sistema renina angiotensina, as plaquetas sanguíneas e eventos trombóticos constatando-se a diminuição da pressão arterial (ZHOU et al., 2014).

Artemisia iwayomogi Kitamura (Compositae) a planta comumente utilizada em alimentos e na medicina tradicional da Coréia e na China. A planta e compostas por componentes bioativos responsáveis por suas atividades farmacológicas, como antialérgicas, anti-inflamatórias, como também efeitos positivos contra doenças 
hepáticas, distúrbios imunes e oxidativos ocasionados pelo estresse (JEON; KIM; LEE, 2014; SHIN et al., 2014). Já a Curcuma longa, popularmente conhecida como açafrão, é uma planta perene pertencente à família Zingiberaceae. É uma planta comumente utilizada para terapias da saúde na Ásia, para diversos tratamentos como cicatrização de feridas e distúrbios inflamatórios, além de ocupar uma posição importante na medicina tradicional da Índia (CHANDRASEKARAN et al., 2013). $\mathrm{Na}$ pesquisa selecionada, o artigo analisou extratos de cada uma destas plantas $A$. iwayomogi e $C$. longa, e a combinação de ambas as plantas, para identificar se a junção das mesmas potencializa efeitos anti-hiperlipidêmicos em ratos induzidos a uma dieta de alta gordura. O resultado obtido foi que tanto os ratos tratados com o extrato de cada planta e a junção das mesmas, atenuou significativamente o perfil lipídico aumentado devido a ingestão de uma dieta elevada de gordura. Contudo, o extrato com ambas as amplas, obteve melhores resultados em relação no nível sérico dos triglicerídeos, também na esteatose hepática e nos níveis de eliminação dos radicais livres se comparado com os extratos utilizando cada planta separadamente (RADDY et al., 2015). Em um outro estudo, que também analisou a junção entre as plantas Artemisia iwayomogy Kitamura e Curcuma longa Linne com o intuito de inibir o acumulo de gordura nas artérias, que geram a placa aterosclerótica, o estudo identificou a diminuição notavelmente não apenas de lesões ateroscleróticas, mas também mediadores ateroscleróticos significativamente normalizados em tecidos séricos ou aórtico, sendo potentes na capacidade antioxidantes, além de atenuar as citosinas pró-inflamatórias, bem como o estresse oxidativo (SHIN et al., 2014).

\section{CONCLUSÕES}

As plantas medicinais possuem potenciais benefícios para o tratamento da hiperlipidemia, principalmente se considerar as baixas taxas de toxicidade que as mesmas apresentam, ocasionando principalmente um importante aliado no tratamento de doenças cardíacas. No entanto, é necessário o desenvolvimento de mais pesquisas científicas para que sejam identificados os inúmeros compostos ativos que as plantas possuem, posto que ainda não estão totalmente elucidados os seus mecanismos de ação, para que seja desenvolvido novos fármacos derivados de produtos naturais.

\section{REFERÊNCIAS}

ANVISA. AGENCIA NACIONAL DE VIGILÂNCIA SANITÁRIA. Farmacopeia Homeopática Brasileira. 3a Ed. Brasília, 2011. Disponível em: <http://www.anvisa.gov.br/hotsite/farmacopeiabrasileira/conteudo/3a_edicao.pd>

ANVISA. AGENCIA NACIONAL DE VIGILÂNCIA SANITÁRIA. Formulário Homeopática: Farmacopeia Brasileira. 1르 Ed. Brasilia,2017. Disponível em: $<$ http://portal.anvisa.gov.br>

ASNAASHARI, M.; HASHEMI, S. M. B.; MEHR, H. M.; YOUSEFABAD, S. H. Kolkhoung (Pistacia khinjuk) Hull Oil and Kernel Oil as Antioxidative Vegetable Oils with High Oxidative Stability and Nutritional Value. Food Technol Biotechnol, v.53, n.1, p. 81-86, 2015. Disponível em: <https://www.ncbi.nlm.nih.gov/pmc/articles/PMC5068431/\#_ffn_sectitle>. doi: 10.17113/ffb.53.01.15.3719 
BRASIL. Ministério da Saúde. MS elabora Relação de Plantas Medicinais de Interesse ao SUS. Agencia Saúde, Brasília: Ministério da Saúde, 2009. Disponível em:

$<$ http://bvsms.saude.gov.br/bvs/sus/pdf/marco/ms_relacao_plantas_medicinais_sus_ 0603.pdf>. Acesso em: 12 jan. 2018

BRASIL. Ministério da Saúde. Secretaria de Ciência, Tecnologia e Insumos Estratégicos. Departamento de Assistência Farmacêutica. Política e Programa Nacional de Plantas Medicinais e Fitoterápicos. Brasília: Ministério da Saúde, 2016.

Disponível em: $<$ http://bvsms.saude.gov.br/bvs/publicacoes/politica_programa_nacional_plantas_me dicinais_fitoterapicos.pdf>. Acesso em: 22 de jan. 2018

CHANDRASEKARAN, C. V.; SUNDARAJAN, K.; EDWIN, J. R.; GURURAJA, G. M.; MUNDKINAJEDDU, D.; et al. Immune-stimulatory and anti-inflammatory activities of Curcuma longa extract and its polysaccharide fraction. Pharmacognosy Research, v. 5, n. 2, p. 71-79, 2013. Disponível em: <https://www.ncbi.nlm.nih.gov/pmc/articles/pmid/23798880/>. doi: 10.4103/09748490.110527.

DE FIGUEIREDO, P. R.; OLIVEIRA, I. B.; NETO, J. B. S.; DE OLIVEIRA J. A.; RIBEIRO L. B.; et al. Caryocar coriaceum Wittm. (Pequi) fixed oil presents hypolipemic and anti-inflammatory effects in vivo and in vitro. Journal of Ethnopharmacology, Ceará - Brasil, v. 191, p. 87-94, 2016. Disponível em: <http://www.sciencedirect.com/science/article/pii/S037887411630397X?via\%3Dihub> . doi: https://doi.org/10.1016/j.jep.2016.06.038

EL-TANTAWY, W. H.; TEMRAZ, A.; HOZAIEN, H. E.; EL-GINDI, O.; TAHA, K. F. Antihyperlipidemic activity of an extract from roots and rhizomes of Panicum repens $L$. on high cholesterol diet-induced hyperlipidemia in rats. Zeitschrift für Naturforschung C, v. 70, n. 5-6, p. 139-44, $2014 . \quad$ Disponível em: <https://www.degruyter.com/view/j/znc.2015.70.issue-5-6/znc-2014-4147/znc-20144147.xml>. doi: 10.1515/20144147

FALUDI, A. A.; IZAR C. O. M.; SARAIVA J. F. K.; CHACRA A. P. M. BIANCO H. T.; et. al. Arquivo brasileiro de cardiologia: atualização da diretriz brasileira de dislipidemias e prevenção da aterosclerose - 2017. Sociedade Brasileira De Cardiologia, v. 109, n. 2 p. $\quad 1-92, \quad 2017.2$ Disponível em: <http://publicacoes.cardiol.br/2014/diretrizes/2017/02_DIRETRIZ_DE_DISLIPIDEMIA S.pdf>. Acesso em: 24 ago. 2017.

FIRMO, W. C. A.; MENEZES, V. J. M; PASSOS, C. E. C; DIAS, C. N.; ALES, L. P. L.; et al. Contexto histórico, uso popular e concepção científica sobre plantas medicinais. Caderno de pesquisa, São Luís, v. 18, n. especial, p. 90-95, 2011. Disponível em: <http://www.periodicoseletronicos.ufma.br/index.php/cadernosdepesquisa/article/vie w/746>. Acesso em: 23 ago. 2017

HAN, J. M.; LEE, J. S.; KIM H. G.; SEOL, I. C.; IM, H. J.; et al. Synergistic effects of Artemisia iwayomogi and Curcuma longa radix on high-fat diet-induced 
hyperlipidemia in a mouse model. Journal of Ethnopharmacology, v. 173, n. 15, p. 217-224, 2015. Disponível em: <https://doi.org/10.1016/j.jep.2015.07.021>. doi: 10.1016/j.jep.2015.07.021

JEON, J. H.; KIM, M. G.; LEE, H. S. Acaricidal activities of bicyclic monoterpene ketones from Artemisia iwayomogi against Dermatophagoides spp. Experimental and Applied Acarology, v. 62, n.3, p. 415-422, 2014. Disponível em: <https://dx.doi.org/10.1007/s10493-013-9739-x>. doi:10.1007/s10493-013-9739-x

JOKAR, A.; MASSOMI, P.; SADEGHPOUR, O.; NASSIRI-TOOSI, M.; HAMEDI , S. Potential therapeutic applications for Terminalia chebula in Iranian traditionalmedicine. Journal of Traditional Chinese Medicina, v. 36, n. 2, p. 250254, 2016. Disponível em: <https://www.ncbi.nlm.nih.gov/pubmed/27400482>. Acesso em: 20 ago. 2017

KAMAL, F.; SHAHZAD, M.; AHMAD, T.; AHMED, Z.; TAREEN, R. B.; et al. Antihyperlipidemic effect of Pistacia khinjuk. Biomedicine \& Pharmacotherapy, v. 96, p. 695-699, 2017. Disponível em: <https://doi.org/10.1016/j.biopha.2017.10.061>. doi: 10.1016/j.biopha.2017.10.061

KAUR, G.; PATEL, R. S. S. Preventive and Curative Potential of Vigna mungo against Metabolic Syndrome in Acute and Chronic Rat Models. Journal of Biological Sciences, v. 15, n. 2, p. 85-91, 2015. Disponível em: <http://scialert.net/abstract/?doi=jbs.2015.85.91>. doi. 10.3923/jbs.2015.85.91

KERNTOPF, M. R.; FIGUEIREDO, P. R. L.; FELIPE, C. F. B.; ALMEIDA, W. O.; MENEZES, I. R. A.; et al. Óleo de pequi (caryocar coriaceum w.) E a potencial atividade cardioprotetor. Ensaios e Ciência: Ciências Biológicas, Agrárias e da Saúde, Campo Grande, v. 3, n. 1, p. 117-125, 2013. Disponível em: <http://www.redalyc.org/articulo.oa?id=26032925010 >. Acesso em: 2 set. 2017

LEGUA, P.; SERRANO, M.; MELGAREJO, P.; VALERO, D.; MARTINEZ, J. J.; et al. Quality parameters, biocompounds and antioxidant activity in fruits of nine quince (Cydonia oblonga Miller) accessions. Scientia Horticulturae, v. 154, p. 61-65, 2013. Disponível em: <https://doi.org/10.1016/j.scienta.2013.02.017>. doi: 10.1016/j.scienta.2013.02.017

NEHDI, I. A.; SBIHI, H.; TAN, P. C.; AL-RESAYES, S. I. Evaluation and characterisation of Citrullus colocynthis (L.) Schrad seed oil: Comparison with Helianthus annuus(sunflower) seed oil. Food Chemistry, v. 136, n. 2, p. 348353, 2013. Disponível em: <https://doi.org/10.1016/j.foodchem.2012.09.009>. doi: 10.1016/j.foodchem.2012.09.009

PEREIRA, J. B. A.; RODRIGUES, M. M.; MORAIS, I. R.; VIEIRA, C. R. S; SAMPAIO, J. P. M.; et al. O papel terapêutico do Programa Farmácia Viva e das plantas medicinais. Revista brasileira de Plantas Medicinais, Botucatu, v. 17, n. 4, p. 550561, 2015. Disponível em: <http://dx.doi.org/10.1590/1983-084X/14_008>. doi: 10.1590/1983-084X/14_008 
RADDY, M. M.; DEVAVARAM, J. D.; DHAS, J.; ADEGHATE, E.; EMERALD, B. S. Anti-hyperlipidemic effect of methanol bark extract of Terminalia chebula in male albino Wistar rats. Journal Pharmaceutical Biology, v.53, n. 8, p. 1133-1140, 2015. Disponível em: <https://doi.org/10.3109/13880209.2014.962058>. doi: 10.3109/13880209.2014.962058

SARABHAI S.; SHARMA, P.; CAPALASH, N. Ellagic acid derivatives from Terminalia chebula Retz. downregulate the expression of quorum sensing genes to attenuate Pseudomonas aeruginosa PAO1 Virulence. Plos ONE, v.8, n.1, p. 1-11, 2013. Disponível em: doi:10.1371/journal.pone.0053441 $<$ https://doi.org/10.1371/journal.pone.0053441>.

SHIN, H. S.; HAN, J. M. ; KIM, H. G.; CHOI, M. K.; FILHO, C. G.; et al. Antiatherosclerosis and hyperlipidemia effects of herbal mixture, Artemisia iwayomogi Kitamura and Curcuma longaLinne, in apolipoprotein E-deficient mice. Journal of Ethnopharmacology, v. 153, n. 1, p. 142-150, 2014. Disponível em: <https://doi.org/10.1016/j.jep.2014.01.039>. doi: 10.1016/j.jep.2014.01.039

SINGH, S. V.; MANHAS, A.; SINGH, S. P.; MISHRA, S. ; TIWARI, N.; et al. A phenolic glycoside from Flacourtia indica induces heme mediated oxidative stress in Plasmodium falciparum and attenuates malaria pathogenesis in mice. Phytomedicine, v. 30, n. 1, p. 1-9, 2017. Disponível em: <https://doi.org/10.1016/j.phymed.2017.04.010>. doi: 10.1016/j.phymed.2017.04.010

SINGH, S.V.; SHRIVASTAVA, A.; JYOTSHNA; CHATURVEDI, L.; SINGH, S. C.; et al. A mechanism-based pharmacological evaluation of efficacy of Flacourtia indica in management of dyslipidemia and oxidative stress in hyperlipidemic rats. Journal of Basic and Clinical Physiology and Pharmacology, v. 27, n. 2, p. 121-129, 2016. Disponível em: <https://doi.org/10.1515/jbcpp-2015-0017>. doi: 10.1515/jbcpp-20150017

SODEIFIAN, G.; GHORBANDOOST, S.; SAJADIAN, S. A; ARDESTANI, N. S. Extraction of oil from Pistacia khinjuk using supercritical carbon dioxide: Experimental and modeling. The Journal of Supercritical Fluids, v. 110, p. 265-274, 2016. Disponível em: <https://doi.org/10.1016/j.supflu.2015.12.004>. doi: 10.1016/j.supflu.2015.12.004

SOLANKI, Y. B.; JAIN, S. M. Antihyperlipidemic activity of Clitoria ternatea and Vigna mungo in rats. Pharmaceutical Biology, Gujarat, India, v. 48, n. 8, p. 915-923, 2010. Disponível em: <https://doi.org/10.3109/13880200903406147>. doi: $10.3109 / 13880200903406147$

TOSATI, F. C.; SCHEFFER-BASSO, S. M. Aspectos morfofisiológicos e bromatológicos do capim-torpedo (Panicum repens L., Poaceae). Biotemas, v. 20, n. 3, p.7-14, 2007. Disponível em: <http://dx.doi.org/10.5007/\%25x>. doi: h10.5007/25x

UMAR, A.; ISKANDAR, L.; AIKEMU, A.; YIMING; W.; ZHOU, W.; et al. Effects of Cydonia oblonga Miller leaf and fruit flavonoids on blood lipids and anti-oxydant potential in hyperlipidemia rat. Journal of Ethnopharmacology, v. 169, n. 1, p. 239- 
243, 2015. Disponível em: <https://doi.org/10.1016/j.jep.2015.04.038>. doi: 10.1016.2015.04.038

VERMA, P. R.; ITANKAR P. R.; ARORA, S. K. Evaluation of antidiabetic antihyperlipidemic and pancreatic regeneration, potential of aerial parts of Clitoria ternatea. Revista Brasileira de Framacognosia, Curitiba, v. 23, n.5, p. 819-829, 2013. Disponível em: <http://dx.doi.org/10.1590/S0102-695X2013000500015>. doi: $10.1590 /$ S0102-695X2013000500015

WANG, Y. C.; LIN, C.C.; LEE, H. I.; YANG, C.; YANG, C. C. Anti-hyperlipidemic activity of spider brake (Pteris multifida) with rats fed a high cholesterol diet. Pharmaceutical Biology, Taiwan, v. 48, n. 2, p. 221-226, 2010. Disponível em: <https://doi.org/10.3109/13880200903085458>. doi: 10.3109/13880200903085458

ZAMANI, M.; RAHIMI, A. O.; MAHDAVI, R.; NIKBAKHSH, M.; JABBARI , M. V.; et al. Assessment of anti-hyperlipidemic effect of Citrullus colocynthis. Revista Brasileira de Framacognosia, João Pessoa, v. 17, n. 4, p. 492-496, 2007. Disponível em: <http://dx.doi.org/10.1590/S0102-695X2007000400003>. doi: 10.1590102$695 \times 2007000400003$

ZANG, Y.; WU, L.; MA, Z; CHENG, J.; LIU, J. Anti-Diabetic, Anti-Oxidant and AntiHyperlipidemic Activities of Flavonoids from Corn Silk on STZ-Induced Diabetic Mice. Molecules, v. 21, n. 7, p. 1-11, 2016. Disponível em: <http://www.mdpi.com/14203049/21/1/7>. doi:10.3390/molecules21010007

ZHOU, W.; ABDURAHMAN, A.; UMAR, A.; ISKANDER L.; ABDUSALAM, E.; et al. Effects of Cydonia oblonga Miller extracts on blood hemostasis, coagulation and fibrinolysis in mice, and experimental thrombosis in rats. Journal of Ethnopharmacology, v. 154, n.1, p. 163-169, 2014. Disponível em: <https://doi.org/10.1016/j.jep.2014.03.056>. doi: 10.1016/j.jep.2014.03.056

ZAGO, L. M. S. Vinte e dois anos de pesquisa sobre plantas medicinais: uma análise cienciométrica. Tecnia, v. 3, n. 1, p. 157-173, 2018. 